\title{
Comments on the upper main-sequence instability domains
}

\author{
A. A. Pamyatnykh $1,2,3$ \\ ${ }^{1}$ Institute of Astronomy, University of Vienna, Türkenschanzstr.17, \\ A-1180 Vienna, Austria \\ ${ }^{2}$ Copernicus Astronomical Center, Bartycka 18, 00-716 Warsaw, Poland \\ ${ }^{3}$ Institute of Astronomy, Russian Academy of Sciences, Pyatnitskaya Str. 48, \\ 109017 Moscow, Russia
}

\begin{abstract}
Some results of theoretical studies of the instability domains in the upper main sequence are outlined. Using the frequency $-\log T_{\text {eff }}$ diagram for models of the Slowly Pulsating B-stars, we show that a recently detected tendency for the coolest SPBs to oscillate with higher frequencies than those for hotter SPB stars, may agree with the theoretical expectations.
\end{abstract}

\section{Introduction}

In this note I will outline some features of the instability domains in the upper main sequence and comment recent observational and theoretical data of other authors which were declared to be in a disagreement with our results or expectations. The numerical results I will present were obtained in Wojtek Dziembowski's group in Warsaw and in Mike Breger's group in Vienna. The note can be considered as an addition to and, partly, shortened exposition of reviews on pulsational instability domains on the main sequence (Pamyatnykh 1999, 2000, 2003 - hereafter, Papers I, II, III, correspondingly).

The stellar models were computed without taking into account the effects of rotation and convective overshooting. An initial hydrogen abundance of $X=0.70$ and a heavy element abundance $Z=0.02$ were assumed, and the heavy element mixture was that of Grevesse \& Noels (1993). In the stellar envelope, the standard mixing-length theory of convection with the mixinglength parameter $\alpha=1.0$ was used. For opacities, we used the most recent 
version of the OPAL data (Iglesias \& Rogers 1996), supplemented with the low-temperature data of Alexander \& Ferguson (1994). Other details of computations are given in Papers I and II.

\section{The updated HR diagram}

In Fig. 1 we present the location of the instability domains in the HR diagram. This plot is an updated version of Fig. 3 in Paper I. The observed domain of $\gamma$ Dor variables is shown in the lower part of the diagram by two approximately vertical lines ( $G$. Handler, private communication). Also the position of stars with solar-like oscillations is shown. The present Sun is located outside the evolutionary track of $1 M_{\odot}$, even slightly to the left of the ZAMS because our standard parameters of the chemical composition and of the convective mixing-length don't fit appropriate solar values.

An important feature of the stability analysis is an extension of the highgravity mode instability domain (the SPB domain) to larger masses and luminosities, as was for the first time mentioned by Pamyatnykh (1998). We discuss this feature in the next subsection. This extension is continuous if we use OP opacities (Seaton 1996, see Paper I). For the SPB domain and its extension, the low temperature boundary coincides with the TAMS due to strong damping of the high-order gravity modes for more evolved models. The TAMS line can be considered also as an effective Red Edge for the $\beta$ Cep domain because of very fast post-MS evolution of massive stars.

Almost all observed $\beta$ Cep and SPB stars lie within the instability domains. However, the $\beta$ Cep variables in NGC 6231 fall outside the instability region. An increase in heavy element abundance may solve this problem. The $\beta$ Cep instability region extends to high luminosities where no observed variables of this type were found, in spite of the fact that the instability strip becomes wider at high luminosities. We have no satisfactory explanation of this discrepancy. It is possible that some additional factors like mass-loss or fast rotation influence the instability at high luminosities. Kiriakidis et al. (1993) noted that the low-amplitude variability of blue supergiants may be connected with the same $\kappa$-mechanism acting in the $Z$ opacity bump region inside the star.

For $\delta$ Sct variables, we chose 329 stars with available uvby $\beta$ photometry from the catalogue of Rodriguez et al. (2000). The photometric data were transformed to effective temperature, $T_{\text {eff }}$, and to luminosity, $L$, in the same way as in Paper II (an older version of the Rodriguez catalogue was used in that paper). Our comparison with the observations is rather illustrative. We do not discuss here the errors of calibrations, the positions of individual stars and we do not try to correct some data using additional information (like distances for the members of open clusters or according to the HIPPARCOS data). For 


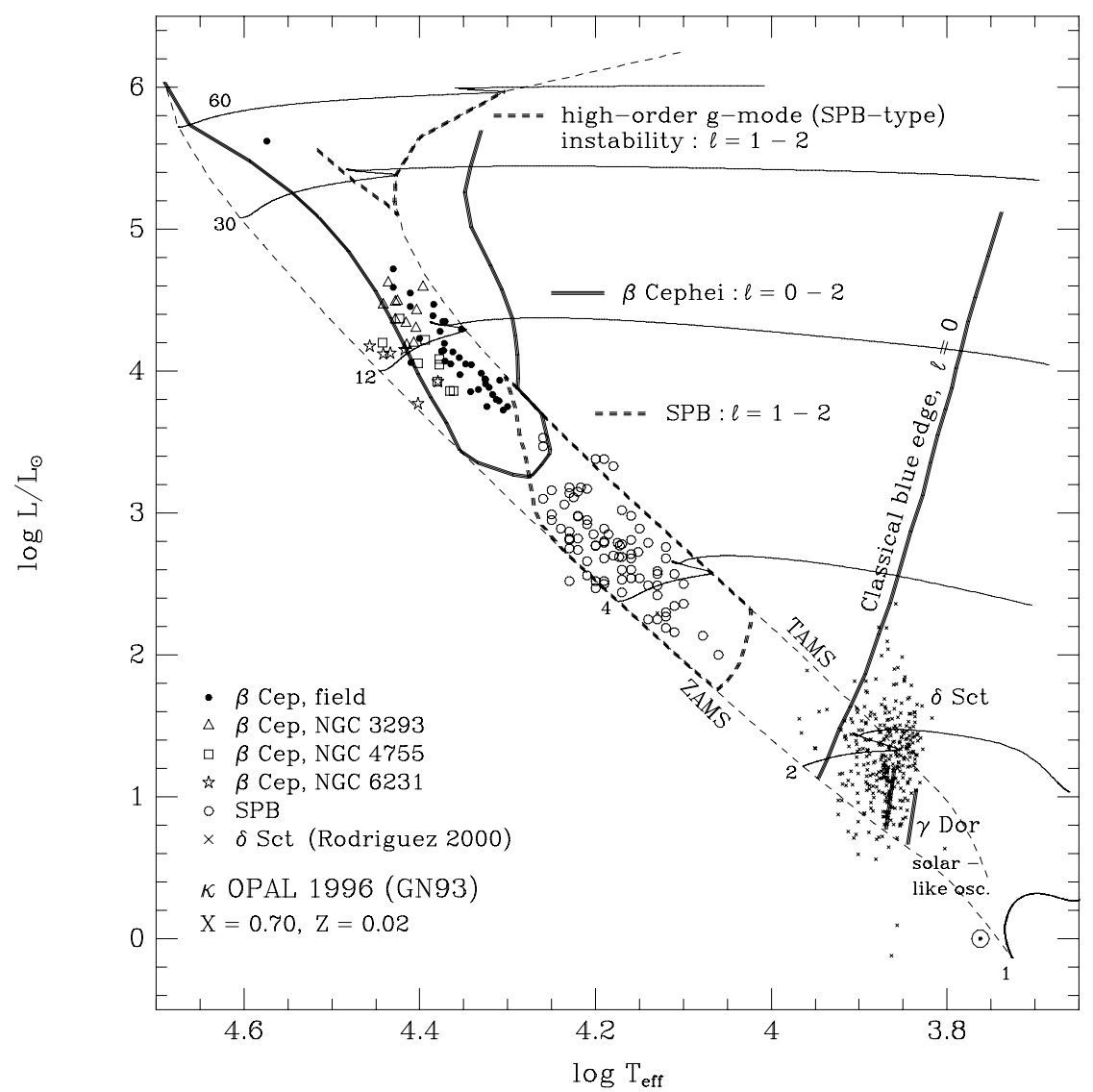

Figure 1: Instability domains in the upper main sequence. Slowly Pulsating B stars are unstable only to nonradial high-order gravity modes. A similar instability was found for massive stars in addition to the $\beta$ Cep-type instability. For the classical instability strip, only the Blue Edge is shown. A few evolutionary tracks for the indicated values of $M / M_{\odot}$ are shown. Observational data are taken from Balona (1994, NGC 3293), Balona \& Koen (1994, NGC 4755), Balona \& Laney (1995, NGC 6231), Sterken \& Jerzykiewicz (1993, $\beta$ Cep in field), North \& Paltani (1994, SPB), Waelkens et al. (1998, SPB discovered by Hipparcos), and Rodriguez et al. (2000, $\delta$ Sct). The location of $\gamma$ Dor variables and of stars with solar-like oscillation is also shown. The position of the present Sun is marked by the symbol $\odot$.

the theoretical instability domain, only the Blue Edge of the instability strip is shown, because the Red Edge is not determined in our computations in which 
we neglected variations of the convective flux during the pulsation cycle. The independence of the driving on the spherical harmonic degree, $\ell$, is a typical feature of modes excited by the $\kappa$-mechanism. Therefore the Blue Edge was computed for radial pulsations. Near the ZAMS, the hottest unstable model is pulsating in the seventh overtone, $p_{8}$, changing to the fourth overtone, $p_{5}$, at $\log L / L_{\odot} \approx 2.0$, and to the fundamental mode, $p_{1}$ at high luminosities, $\log L / L_{\odot} \approx 5.0$.

Recently, Deng \& Xiong (2001) also determined the instability domain of the $\beta$ Cep variables. There exists a significant quantitative disagreement of their results with ours. For example, for a standard heavy element abundance $(Z=0.02)$ they did not find any instability on the main sequence for stellar masses larger than, approximately, $20 M_{\odot}$. In all our computations (Fig. 1, Paper I, see also earlier studies by Moskalik \& Dziembowski 1992, Dziembowski \& Pamyatnykh 1993) no upper limit for the instability was found in the studied mass range of $M \leq 40 M_{\odot}$.

The disagreement may be due to the fact that Deng \& Xiong used an analytical approximation to the opacity tables whereas we used elaborate subroutines of spline interpolation in the tables. We used new versions of the OPAL and OP tables. These tables are fine-zoned in $\log T$ and in $\log R=\log \rho / T_{6}^{3}$ where $T_{6} \equiv T / 10^{6}$ (additionally, we used even more fine-zoned tables which were constructed using spline interpolation). Together with the opacity tables, we used beforehand prepared tables for the opacity derivatives, these tables were constructed also with using the splines. We performed a lot of tests to guarantee the smoothness of the opacity and the derivatives. We are not sure whether an analytical approximation for the opacity can give more reliable results on the instability than our results, because even a good approximation for the opacity may not guarantee the adequate representation of the opacity derivatives which are most important in the instability studies (see Paper I).

Recently, Pigulski \& Kolaczkowski (2002) found a high-luminosity $\beta$ Cep variable in the LMC which may be inconsistent with results by Deng \& Xiong. Moreover, variability of some Luminous Blue Variables can be also caused by pulsation (see, for example, Dorfi \& Gautschy 2002).

The extension of high-gravity mode instability domain

Let's consider the instability of massive stars in more detail. There are two distinct ranges of unstable periods for these models. Shorter periods correspond to low-order mode ( $\beta$ Cep-type) instability, longer periods correspond to an extension of the SPB-type instability. Such a high-gravity mode instability occurs only in the evolved MS-models close to the TAMS, as can be seen from Fig. 1. 

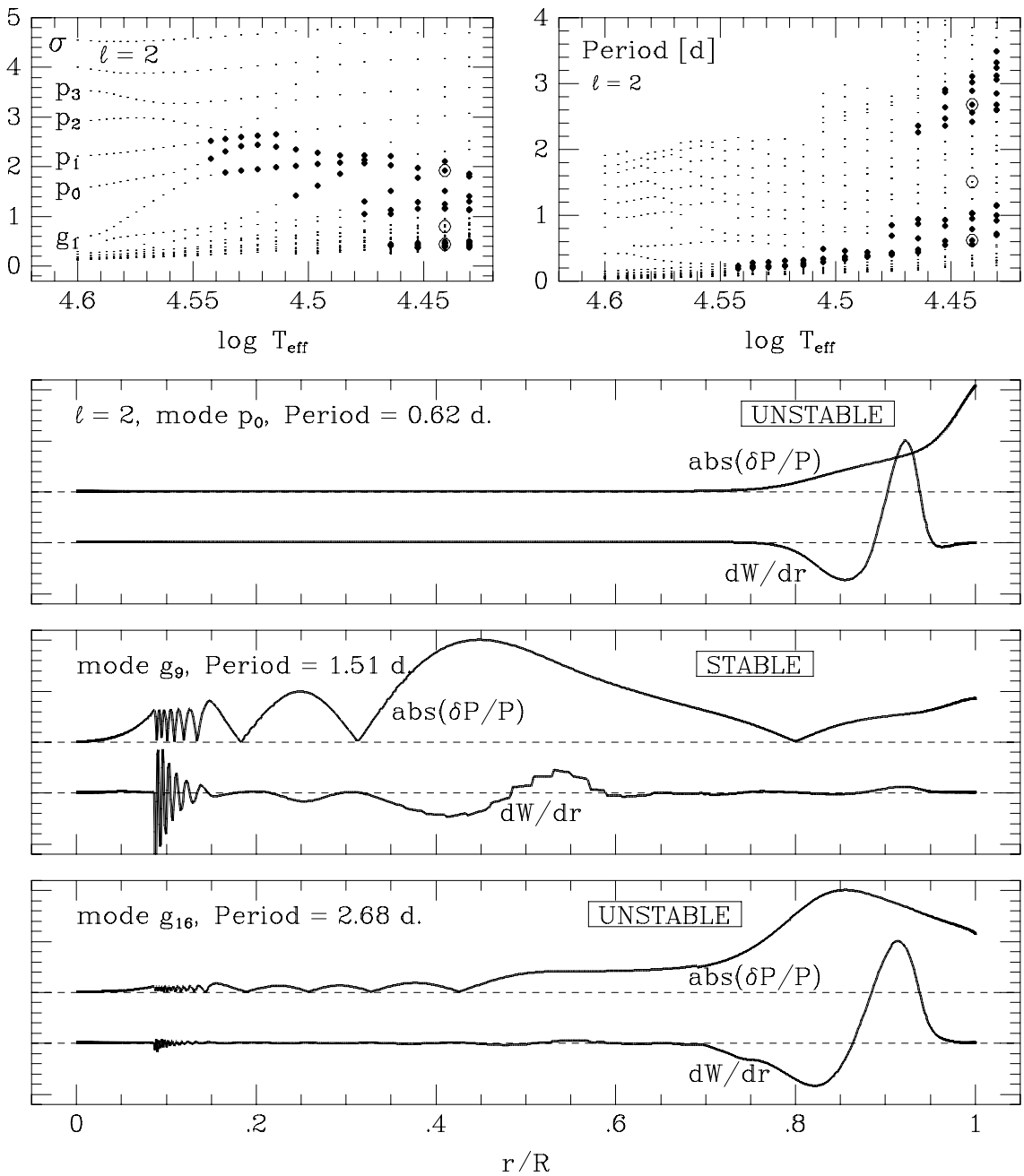

Figure 2: Upper panels: Dimensionless frequencies, $\sigma=\omega / \sqrt{4 \pi G\langle\rho\rangle}$, and periods of quadrupole modes for a $30 M_{\odot}$ star in its MS evolution (from the left to the right). The small and large dots correspond to stable and unstable modes, respectively. Open circles at $\log T_{\text {eff }} \approx 4.44$ mark modes shown in the lower panels.

Lower panels: The differential work integral, $d W / d r$, and the pressure eigenfunction, $\delta P / P$ (both in arbitrary units), for three selected modes. Zero-lines for the variables are shifted with respect to each other. The metal opacity bump is located at $r / R \approx 0.916$ (from Pamyatnykh 1999). 
In Fig. 2, the behavior of the eigenfunction $\delta P / P$ and of the work integral in three quadrupole modes of a $30 M_{\odot}$ model near the TAMS is shown. The evolution of the frequency spectrum from the ZAMS to the TAMS is shown in the upper panels. Three modes chosen for analysis are marked by open circles.

The mode with a period of 0.62 days is formally the $g_{3}$-mode according to the evolution of the frequency spectrum, see upper left panel $(\sigma \approx 2$ for this mode). But during the evolution from the ZAMS a coupling between this mode $g_{3}$ and the mode $p_{0}$ occurred via an avoided crossing. Therefore, this mode is now similar to the acoustic mode $p_{0}$ according to it's kinetic energy, which is confined mainly in the envelope. The behavior of the pressure eigenfunction also mimics that of the lowest-order acoustic mode. That is why we classify this mode as $p_{0}$. The opacity mechanism will work effectively if, in the driving region, $\delta P / P$ is large and does not vary rapidly. That is true both for $p_{0}$ and for $g_{16}$. At intermediate periods the amplitude of the pressure variations is relatively small in the potentially driving region, so the damping in the interiors exceeds the driving in the envelope.

\section{Period $-\log T_{\text {eff }}$ and Frequency $-\log T_{\text {eff }}$ diagrams}

In Fig. 3, the periods of unstable modes are shown separately for $\ell=0,1$ and 2. This figure is similar to Fig. 6 in Paper II, only the OPAL opacities have been used in the present case. Note two distinct ranges of unstable periods for massive stars. For clarity, the unstable modes of the MS models with masses 4 and $12 M_{\odot}$ are marked by heavy symbols. Here, the leftmost points correspond to the ZAMS model, and the rightmost ones - to the TAMS model. For the ZAMS $4 M_{\odot}$ model, the high-order gravity modes $g_{9}$ to $g_{16}$ of $\ell=1$ and $g_{9}$ to $g_{21}$ of $\ell=2$ are excited. For the TAMS $4 M_{\odot}$ model, the high-order gravity modes $g_{36}$ to $g_{81}$ of $\ell=1$ and $g_{43}$ to $g_{85}$ of $\ell=2$ are excited (see Fig. 2 in Paper III for more details). The jagged appearance of the instability domains is due to considering discrete values of stellar masses.

Quite recently, Peter De Cat \& Conny Aerts published very interesting results of a study of bright southern SPB stars (De Cat \& Aerts 2002). They found that "the observed frequencies of the coolest SPB stars tend to be higher than those of the hotter stars, although the trend is only marginal" (see Fig. 18 in their paper). In the author's opinion, "such a temperature-frequency relation is not expected from theoretical excitation studies". However, we are not sure, whether such a disagreement between theory and observations does exist.

In Fig. 4 we plot frequencies of unstable modes versus effective temperature both for $\beta$ Cep and SPB type variables. Nonradial acoustic modes in the $\beta$ Cep domain are not shown, their frequencies lie outside the corresponding boxes because for $\ell=1$ and $\ell=2$ we used frequency range similar to that in 

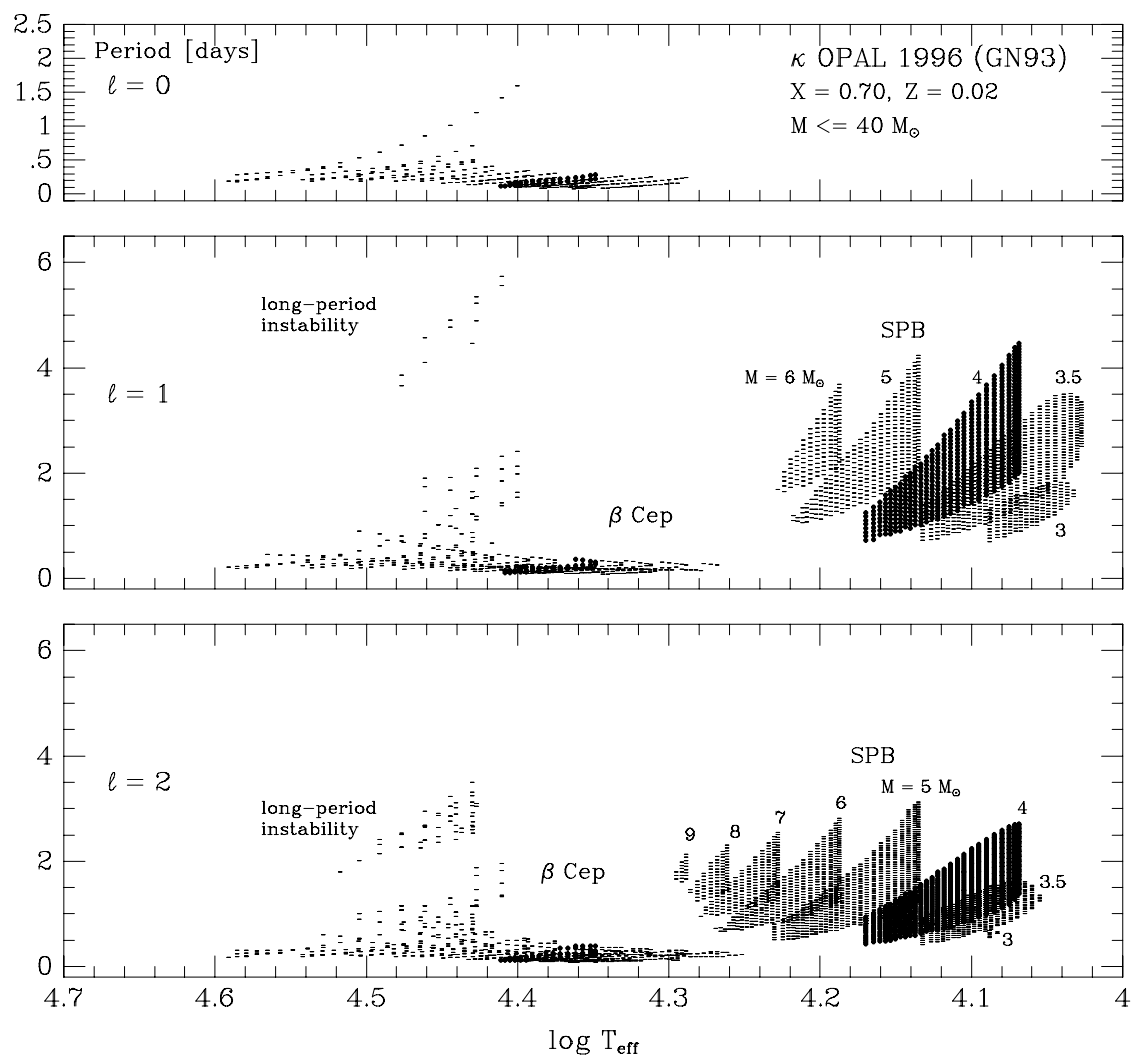

Figure 3: Periods of unstable modes in $\beta$ Cep and SPB domains shown in Fig. 1. For the clarity, the results for two fixed values of stellar mass are marked by heavy symbols. Left and right edges of $4 M_{\odot}$ sequence correspond to the model on ZAMS and TAMS, correspondingly. Note two distinct ranges of unstable periods for massive stars. Shorter periods correspond to low-order mode ( $\beta$ Cep-type) instability, long periods correspond to extension of the SPB-type instability.

Fig. 18 in De Cat \& Aerts (2002). The observed domain from that figure is approximated by thin solid lines.

Indeed, for a given stellar mass, say, $4 M_{\odot}$, unstable frequencies of the cooler models tend to be lower than those of the hotter models. This is because the local thermal time-scale in the driving zone at the metal opacity bump is larger for cooler models (and optimal conditions for excitation of oscillations are fulfilled for longer periods, i.e. for lower frequencies). This tendency is 

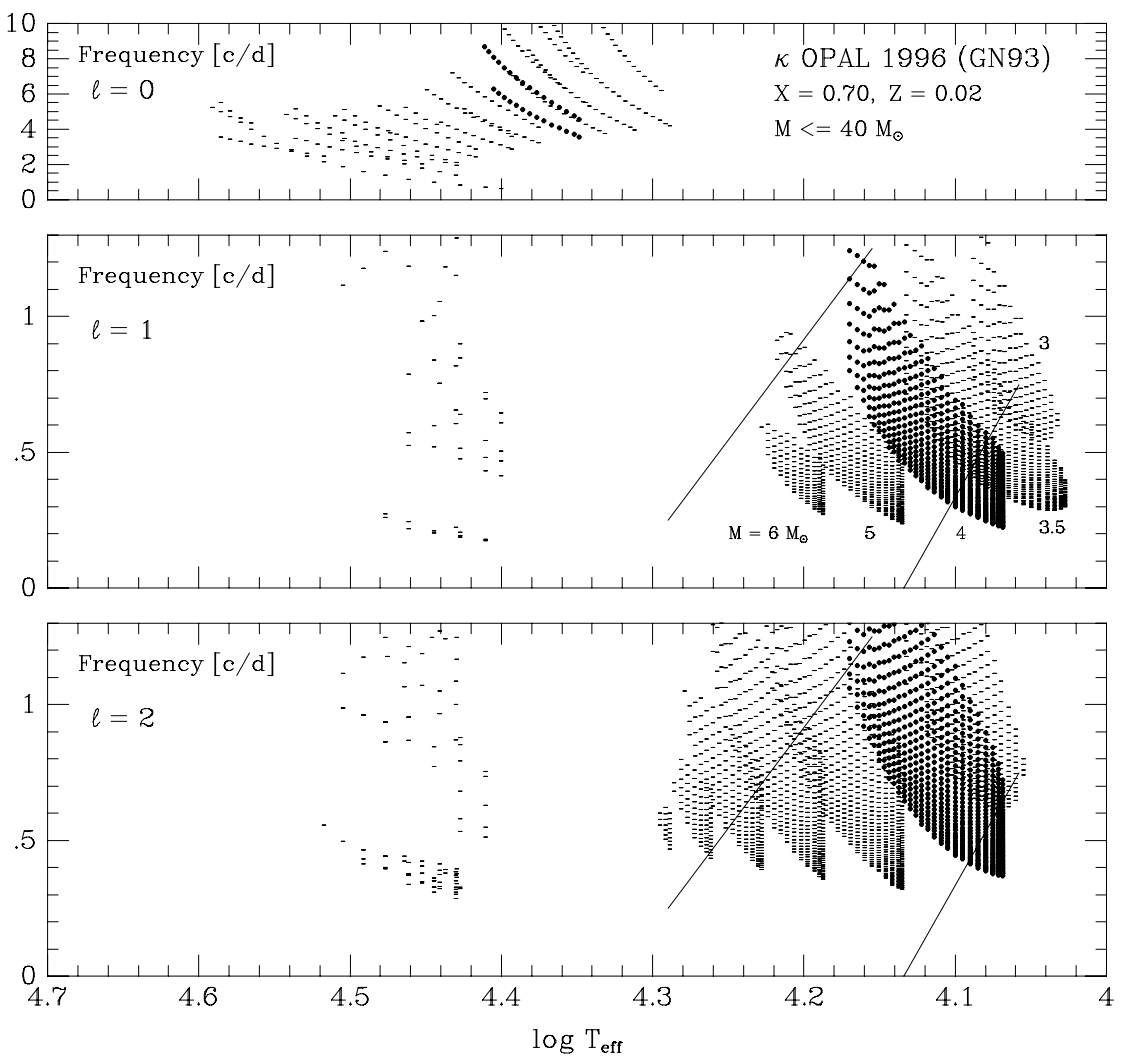

Figure 4: Frequencies of unstable modes in $\beta$ Cep and SPB domains. The same data as in Fig. 3 are used. Nonradial acoustic high-frequency modes in the $\beta$ Cep domain are not shown, their frequencies lie outside the corresponding boxes. Thin solid lines approximate the observed domain of bright SPB stars (Fig. 18 in De Cat \& Aerts 2002).

opposite to the trend found by De Cat \& Aerts. However, for different masses inside the SPB domain along the main sequence, we have a more complicate interplay between opacity and requirements concerning both the shape of the eigenfunctions and the ratio of oscillation period to thermal time-scale. As a consequence, the above mentioned tendency does not exist in this relatively narrow range of periods or frequencies in the SPB domain, as can be seen in Fig. 4. Moreover, for $\ell=1$ we have the same marginal trend as it is observed, and the observed and theoretical domains are in a quite good agreement. The 
only problem may arise for the lowest observed frequencies (longest periods) - all models are stable at these frequencies. It is possible that models with convective overshooting may solve this problem too - if in the HR diagram these stars are located slightly to the right of the MS band computed without overshooting.

To conclude, at present we don't see any significant disagreement between observed results for the SPB variables and theoretical expectations. It is possible, that a disagreement still exists. However, for such a suggestion it is necessary to compare the observed data and theoretical results in more details, taking into account exact location of the stars inside the SBB domain in the HR diagram.

Acknowledgments. This note was written during my stay at the Institute of Astronomy of the University Vienna. I would like to thank Mike Breger and all members of his group for fruitful discussions. All numerical results presented here were obtained in the Wojtek Dziembowski's group in Warsaw and in the Mike Breger's group in Vienna. The investigation has been partly supported by Polish KBN grant No. 5 P03D 01220 and by the Austrian Fonds zur Förderung der wissenschaftlichen Forschung under project number P14546-PHY.

\section{References}

Alexander, D. R., Ferguson, J. W. 1994, ApJ 437, 879

Balona, L. A. 1994, MNRAS 267, 1060

Balona, L. A., Koen, C. 1994, MNRAS 267, 1071

Balona, L. A., Laney, C. D. 1995, MNRAS 276, 627

De Cat, P., Aerts, C. 2002, A\&A 393, 965

Deng, L., Xiong, D. R. 2001, MNRAS 327, 881

Dorfi, E. A., Gautschy, A. 2002, Comm. in Asteroseismology, 141,57

Dziembowski, W. A., Moskalik, P., Pamyatnykh, A. A. 1993, MNRAS 265, 588

Dziembowski, W. A., Pamyatnykh, A. A. 1993, MNRAS 262, 204

Grevesse, N., Noels, A. 1993, in Origin and Evolution of the Elements, eds.

Pratzo N., Vangioni-Flam E., Casse M., Cambridge Univ. Press, p. 15

Iglesias, C. A., Rogers, F. J. 1996, ApJ 464, 943

Kiriakidis, M., Fricke, K. J., Glatzel, W. 1993, MNRAS 264, 50

Moskalik, P., Dziembowski, W. A. 1992, A\&A 256, L5

North, P., Paltani, S. 1994, A\&A 288, 155

Pamyatnykh, A. A. 1998, in A Half-Century of Stellar Pulsation Interpretations, eds. Bradley P. A., Guzik J. A., ASP Conf. Ser., vol. 135, p. 268

Pamyatnykh, A. A. 1999, Acta Astr. 49, 119 (Paper I)

Pamyatnykh, A. A. 2000, in Delta Scuti and Related Stars, eds. Breger M., Montgomery M. H., ASP Conf. Ser., Vol. 210, p. 215 (Paper II)

Pamyatnykh, A. A. 2003, in Asteroseismology Across the HR Diagram, eds. 
Thompson M. J., Cunha M., Monteiro M. J. P. F. G., Kluwer, in press (Paper III) Pigulski, A., Kołaczkowski, Z. 2002, A\&A 388, 88

Rodriguez, E., López-González, M. J., López de Coca, P. 2000, in Delta Scuti and Related Stars, eds. Breger M., Montgomery M. H., ASP Conf. Ser., Vol. 210, p. 499

Seaton, M. J. 1996, MNRAS 279, 95

Sterken, C., Jerzykiewicz, M. 1993, Space Sci. Rev. 62, 95

Waelkens, C. 1991, A\&A 246, 453

Waelkens, C., Aerts C., Kestens E., Grenon M., Eyer L. 1998, A\&A 330, 215 